Editorial

\title{
Journal of neurology \& stroke: overview about the future of vascular neurology
}

Volume I Issue 4 - 2014

\section{Editorial}

Nowadays, in several parts of the globe, chronic and acute cerebrovascular diseases, account for an important component in the rate of mortality and morbidity that largely reaches an economically active population.

The importance of clinical trials and basic area studies, may contribute to the development in the diagnosis and treatment of these diseases and more often than ever, stroke has been emphasized as priority in several treatment centers of the central nervous system diseases worldwide.

The Journal of Neurology \& Stroke is a vehicle of fantastic quality to divulge scientific researches in basic and clinical areas in the field of vascular neurology.

Aspects that concern the genetic and molecular biology of vascular malformations and brain diseases will have a prominent place, to the extent that the articles of an experimental nature and basic research are evaluated.

Neurochemistry and pathways of metabolism that involve the steps of pathophysiology of certain cerebrovascular disorders and the occlusive effect of these conditions are fundamental to support the bases for understanding these diseases.

There will be without any doubt a place for quality articles in the area of neurosurgery showing ways and techniques that could assist in the treatment of occlusive cerebrovascular diseases, spinal cord and encephalic arteriovenous malformations cerebral aneurysms, cavernomas, dural fistulas, cerebral and cerebellar hemorrhage and cerebellar moyamoya disease and techniques to perform cerebral arterial encephalic bypass.

Endovascular treatment should not and would not remain outside of that sphere of importance in the treatment of vascular aneurysms, arterial encephalic occlusions, arteriovenous dural fistulas and other arterial venous malformations. New technologies will have a place to be published since meet the required ethical aspects and formalities of each clinical institute and country of research.

\author{
Paulo Henrique Pires de Aguiar \\ Department of Neurosurgery, Federal University of Rio Grande \\ do Sul, Brazil
}

Correspondence: Paulo Henrique Pires de Aguiar, Department of Neurosurgery, Santa Paula Hospital, R. Barata Ribeiro, 237 - Bela Vista, São Paulo - SP, I 38-, Brazil, Email phpaenurocir@gmail.com

Received:September 0I, 2014 | Published: September 02, 2014

The trials of venous thrombolysis and brain arterial occlusive diseases gained prominence as well as the endovascular treatments. Nanotechnology is also appreciated in the study and treatment of these diseases.

Aspects of diagnosis by imaging and cerebral hemodynamic studied by transcranial doppler undoubtedly are attaining substratum and have renowned publishers that can bring information to the entire scientific community in the study of cerebral vasospasm post subarachnoid hemorrhage and in the neuro-intensive treatment of vascular diseases in critical condition patients.

The preeminent international editors from all continents that are involved in the treatment and diagnosis of the central vascular nervous system diseases will be the support for us to be able to orient the publication of this journal.

\section{Acknowledgments}

None.

\section{Conflicts of interest}

None. 